\title{
Psychological Sources of Ambiguity Avoidance
}

\author{
Shawn P. Curley, J. Frank Yates, and Richard A. Abrams \\ University of Michigan
}

\begin{abstract}
Ambiguity is characterized as uncertainty about the probabilities with which outcomes can occur. Previous research has established that subjects, when given a choice between two options differing in their degree of ambiguity, tend to prefer the less ambiguous option, exhibiting ambiguity avoidance. The present paper addresses the psychological sources of this behavior. Five plausible hypotheses for the basis of ambiguity avoidance were extracted from the literature, along with a sixth proposal which questioned the deliberateness of the bchavior. Nonc of the hypotheses had previously been sufficiently examined empirically. In a series of five experiments, each of the proposed explanations of ambiguity avoidance was tested. Of the six, only one, termed "other-evaluation," had an effect on subjects' choice behavior in an ambiguous situation involving monetary lotteries. The other-evaluation hypothesis states that a decision maker, in making a choice, anticipates that others will evaluate his or her decision; and, so, makes the choice that is perceived to be most justifiable to others. This choice is for the option having the smallest degree of ambiguity. It is concluded that the other-evaluation hypothesis offers the most promising direction for future research regarding the psychology of choice under ambiguity. 1986 Academic Press, Inc.
\end{abstract}

Suppose a patient must decide between two potential treatments. Treatment A has been used extensively, and there is substantial information that indicates its success rate to be $50 \%$. Treatment $B$ is new, and there is little information underlying the physician's best guess that its chance of success is $50 \%$. This situation, based upon an example first described by Keynes (1921), and again by Ellsberg (1961), demonstrates that two distinct types of uncertainty can be differentiated. The first type, present in both treatments, is uncertainty as to which outcome will occur: success or no success. The second type of uncertainty, present only in Treatment $\mathbf{B}$, is uncertainty about the success probability itself, and is termed ambiguity (Ellsberg, 1961).

It has been conjectured that, given a choice between the two treat-

The authors thank Rubert Pachella, Donald Fisher, and John Kounios for their assistance in recruiting subjects for Experiments 2 and 5. We are also grateful to Robert Revnew for his assistance in carrying out Experiment 4 . This research was supported in part by National Institute of Mental Health Training Grant MH16892. Richard A. Abrams is now at Washington University, St. Louis, MO. Reprint requests should be sent to Shawn P. Curley, Department of Management Sciences, University of Minnesota, 271 19th Avenue South, Minneapolis, MN 55455. 
ments, many people would prefer Treatment A, exhibiting ambiguity avoidance. This claim, that individuals respond to ambiguity, rather than simply ignore it, has received substantial empirical support in a variety of contexts: choices among monetary lotteries (Becker \& Brownson, 1964; Curley \& Yates, 1985; Slovic \& Tversky, 1974; Yates \& Zukowski, 1976), foreign investment choices (MacCrimmon, 1968), clinical treatment choices (Curley, Eraker, \& Yates, 1984; Hamm \& Bursztajn, 1979), almanac-type questions (Goldsmith \& Sahlin, 1982), judgments of likelihood based on inference (Einhorn \& Hogarth, 1985), and insurance decisions (Hogarth \& Kunreuther, 1984, 1985).

What has not been demonstrated is what underlies the observed behavior: Specifically, why is ambiguity avoided? What prompts or motivates subjects to avoid ambiguity? It is to this question that the present research was addressed. The significance of the issue lies both in the prevalence of ambiguity in real-life decision situations and in the paucity of empirical work in this area.

Clearly, ambiguity is present in many decision situations. In real life, we rarely know what the outcome probabilities are. A better understanding of ambiguity and ambiguity reactions would be a valuable bridge between the theory and application of decision research. Yet, current empirical knowledge regarding this question is sparse. Although lacking empirical foundations, our efforts did not lack background altogether. The literature is replete with hypothesized sources of ambiguity avoidance which, although not empirically substantiated, are theoretically and/or intuitively reasonable. Specifically, five categories of proposed explanations are identifiable in the literature. We now discuss each hypothesis in turn. Included with each hypothesis is a list of references in which the hypothesis was proposed; unless indicated, these proposals were not accompanied by empirical support.

1. Hostile nature (Yates \& Zukowski, 1975). The hostile nature hypothesis conjectures that subjects perceive that the process by which the outcomes are determined is nonrandom for the ambiguous option. Instead, the outcome is perceived to be the result of a process which is antagonistic, or at least competitive, toward themselves. The less favorable outcome in the ambiguous situation is perceived as more likely than the more favorable outcome, and thus the ambiguous option is avoided.

Related hypotheses are that subjects reason that it is better to have more information than less (Roberts, 1963), or that subjects perceive the situation as one over which they have little control (Langer, 1975). The rationale is that, when information is hidden or when control of the situation is out of one's hands, conditions are typically biased against one. It is possible, then, that subjects perceive the ambiguous option as biased against them and therefore to be avoided.

2. Other-evaluation (Ellsberg, 1963; Fellner, 1961; Gardenfors, 1979: 
Knight, 1921; Roberts, 1963; Toda \& Shuford, 1965). In making a decision under ambiguity, the decision maker might anticipate that the decision will be evaluated by others. According to the other-evaluation hypothesis, a choice is made which the decision maker perceives as most justifiable to those others who will evaluate the decision. This hypothesis is similar in nature to that proposed by Slovic (1975) and colleagues (Slovic, Fischhoff, \& Lichtenstein, 1982) in the context of risky choice. The anticipated evaluation by others may be explicit, in that the decision maker is responsible to someone else, whether a superior, peers, or otherwise; or the anticipated evaluation may be implicit, in that others will be observing the decision, and the decision maker desires to appear competent.

3. Self-evaluation (Ellsberg, 1963; Hamm \& Bursztajn, 1979; Roberts, 1963; Toda \& Shuford, 1965). The self-evaluation hypothesis is similar to the other-evaluation hypothesis. Instead of anticipating an evaluation by others, the decision maker anticipates the future self-evaluation of the decision. This approach is a recognition by the decision maker of the "strategy of evaluating his past decisions in order to improve his future decisions" (Toda \& Shuford, 1965, p. 247). The notion is related to the concepts of choice regret and anxiety (Bell, 1982, 1983), in that these reactions result from the evaluation of one's own decisions, and are affected by anticipated feedback.

4. Forced choice (Roberts, 1963). The forced-choice hypothesis states that the less ambiguous option is selected only when all other considerations are equal. Ambiguity is a second dimension in a lexicographic decision rule which is used since the task is a forced choice; however, the decision maker would not actually pay to obtain the chosen option over the unchosen one.

Both Becker and Brownson (1964) and Yates and Zukowski (1976) presented contradictory evidence for this hypothesis by obtaining cash equivalents for ambiguous and nonambiguous gambles. All of these gambles were constructed to have an equal subjective probability .50 of success or no success. Both studies found that the mean cash equivalent value given to the more ambiguous lottery was less than that given to the less ambiguous lottery. This result strongly suggests that subjects would indeed pay to receive their preferred option, in contradiction of the forced-choice hypothesis. One difficulty in interpreting the results of these studies is that the procedure of eliciting cash equivalents that was used in both studies is a pricing task and may not be generalizable to the choice situation. Several studies have shown that different preferences sometimes obtain, depending on whether a choice task or a pricing task is used (Goldstein, 1984; Grether \& Plott, 1979; Lichtenstein \& Slovic, 1971).

5. Uncertainty avoidance. The uncertainty avoidance hypothesis states 
that ambiguity avoidance is a consequence of a more general attitude of uncertainty avoidance. Although not fully explanatory, this hypothesis attempts at least to clarify the behavior itself. Accordingly, this hypothesis would predict that those who avoid ambiguity should also exhibit an avoidance of other aspects of uncertainty. For example, risk aversion, the preference for a guaranteed amount over an uncertain gamble with equal expectation, should correlate with the avoidance of ambiguity. An example of a common mechanism underlying uncertainty reactions is that of a utility, or disutility, for gambling. This concept has been applied to risk-avoiding and risk-seeking behaviors (Fellner, 1961; Fishburn, 1980); and may operate in the ambiguous situation, also.

Other explanations. Another hypothesis regarding ambiguity avoidance arises from an ongoing debate concerning the normativeness of any type of reaction to ambiguity. This debate can be briefly outlined as follows. Ellsberg (1961) demonstrated that any reaction to ambiguity, whether ambiguity avoiding or ambiguity seeking, is inconsistent with even a qualitative probability measure of uncertainty. Consequently, Ellsberg, as did Knight (1921), questioned the adequacy of probability as a measure of the totality of decision uncertainty. This stance clearly contradicts the normative status of probability theory implicit in statistical decision theory (DeGroot, 1970; Winkler, 1972), as based upon expected utility theory (Luce \& Raiffa, 1957; von Neumann \& Morgenstern, 1953). or the more general subjective expected utility theory (Savage, 1972).

In response to this criticism, proponents of statistical decision theory advanced a sixth proposal, which is to question the relevance of ambiguity avoidance (Hamm \& Bursztajn, 1979; Roberts, 1963; Savage, 1972). According to this "mistake hypothesis," in expressing a preference for the less ambiguous option, subjects are making a nonconscious, systematic error which, if sufficiently understood, they would correct. This argument is similar to that advanced in support of transitivity in preference patterns. Subjects typically do wish to correct their responses so as to remove intransitivities, which they exhibit with certain elicitation procedures, when these intransitivities are identified to them (MacCrimmon, 1968; Tversky, 1969).

Underlying the mistake hypothesis are the various "Dutch book" arguments which have been advanced by proponents of statistical decision theory (Raiffa, 1961; Roberts, 1963; Savage, 1972; Winkler, 1972). A Dutch book is a series of bets in which the person is sure to lose because his or her beliefs or preferences violate a certain principle. If such is the case, the person is clearly behaving irrationally and should correct the error. Several authors have demonstrated how Dutch books can be constructed at the expense of individuals whose probability judgments are 
not additive (Winkler, 1972), or who exhibit the conjunction fallacy (Tversky \& Kahneman, 1983), or whose probability judgment revisions are non-Bayesian (Freedman \& Purves, 1969). However, as argued in Fellner (1961), a Dutch book based on ambiguity preferences, without arguable assumptions, has not yet been advanced. The reluctance of subjects to alter their ambiguity-avoiding behavior after the presentation of prescriptive counterarguments (MacCrimmon, 1968; MacCrimmon \& Larsson, 1979; Slovic \& Tversky, 1974) further weakens the claim that, upon reflection, people consider ambiguity preferences to be mistakes.

Finally, in addition to these proposed explanations of ambiguity avoidance, there exist a variety of comments which were meant as merely illustrative, rather than psychologically informative. For example, it has been stated that ambiguity leads to low confidence (Becker \& Brownson, 1964; Knight, 1921); however, this is not offered as an explanation, and should not be so interpreted. Such a comment helps illustrate ambiguity reactions without answering the question: Why should this be so? Similar statements are that an ambiguous option has a more volatile probability of success (Ellsberg, 1961; Roberts, 1963) or that ambiguity is avoided when the outcomes at stake are of importance (Fellner, 1961; Hamm \& Bursztajn, 1979). Propositions such as these tend to be tautological in content, serving as attempts to clarify the definitions, stating that an ambiguous option is avoided because it is more uncertain. The comments are not sufficient as explanations, and should not be interpreted as explanatory.

Returning then to the hypothesized explanations of ambiguity avoidance, our intent was to investigate the proposals. As we have pointed out, the proposals have all been advanced as sources of ambiguity avoidance; and, as we have argued, each is a reasonable explanation of the phenomenon. Still, the hypotheses have been largely untested. Our goal was to derive necessary implications for each hypothesis, and then to empirically determine the validity of these implications. To the extent that the implications are not verified, doubt is cast upon the hypotheses having those implications. Naturally, we would not expect that all the explanations would hold. That is to say that the null hypothesis, that an explanation is not valid, was not expected to be rejected in all cases. These null results are included as important evidence regarding the inadequacy of the associated proposed explanations of ambiguity avoidance.

The remainder of this paper presents, and discusses the implications of, the experiments that were designed to illuminate the question, "Why is ambiguity avoided?" We begin by describing the choice situation that was used to test the proposed explanations, and then present five experiments in detail. Experiments 1-4 considered the five plausible explana- 
tions of ambiguity avoidance; Experiment 5 tested the viability of the mistake hypothesis.

\section{GENERAL PROCEDURE}

To test the hypotheses, subjects were asked to evaluate, and indicate their preference between, two lotteries. The two lotteries were essentially the same for each experiment, and are described here in the form used for Experiment 1. Changes in the lotteries within the other studies are noted where appropriate. Each lottery involved risk, in that two outcomes were possible - a player might win $\$ 5$ or nothing. The lotteries differed in their degree of ambiguity. For one, the player knew precisely what the probabilities of each outcome were. For the other, the probabilities were not precisely specified; the lottery was ambiguous. The lotteries were adapted from ones first introduced by Keynes (1921) and later elaborated by Ellsberg (1961). They are illustrated in Fig. 1 and worked as follows.

For each lottery the player was to select a poker chip from a bag without looking. The nonambiguous Lottery 1 bag had a known composition of 50 red and 50 white poker chips; the ambiguous Lottery 2 bag contained $100 \mathrm{red}$ and/or white chips in an unspecified distribution. The player was allowed to name the "valuable" chip color, either red or white, draw one chip from the bag without looking, and would win $\$ 5$ if the selected chip was valuable, and nothing otherwise. For all experiments, the poker chips, bags, and some form of display, like that in Fig. 1 , were in full view of the subjects when the lotteries were explained.

Since the two lotteries were otherwise identical, any differences in a subject's evaluation of the lotteries could be attributed to the difference in their ambiguity. Thus, the degree of ambiguity avoidance could be operationalized as the degree to which subjects preferred the nonambiguous Lottery 1 over the ambiguous Lottery 2 . This operational variable was related to various dependent variables in the experiments, in tests of necessary implications of each hypothesis.

\begin{tabular}{|c|c|}
\hline Lottery 1 & Lottery 2 \\
\hline $\begin{array}{l}50 \text { Red chips } \\
50 \text { White chips } \\
100 \text { Totd }\end{array}$ & $\begin{array}{l}? \text { Red chips } \\
? \text { White chips } \\
100 \text { Total }\end{array}$ \\
\hline
\end{tabular}

FIG. 1. Schematic descriptions of Lottery 1, Lottery 2, and the playing procedure of each. 


\section{EXPERIMENT 1}

Experiment 1 was designed to study the relationship between preferences for risk and ambiguity in order to determine the validity of the uncertainty avoidance hypothesis (Hypothesis 5). If a common mechanism underlies responses to risk and ambiguity, an individual's response to the uncertainty of risk, i.e., uncertainty as to which outcome will occur, should be related to his or her response to the additional uncertainty present in an ambiguous option. For example, a person who avoids the uncertainty of risk would be expected to also avoid the uncertainty of ambiguity. Lotteries 1 and 2 (Fig. 1) were the lotteries used in the study.

\section{Method}

Subjects. Twenty-six University of Michigan undergraduates enrolled in an introductory psychology course served as subjects. The experimental task was presented to them in a single group session.

Procedure. Following a verbal explanation of Lottery 1, a cash equivalent was obtained for each subject, using a procedure introduced by Becker, DeGroot, and Marschak (1964). The cash equivalent was described as the smallest amount of money the subject would accept in exchange for the opportunity to play the lottery in his or her possession, that is, a minimum selling price for Lottery 1 . Also indicated was that, after the subject stated this price, the experimenter would offer the subject a buying price. The buying price would be randomly selected from a bag containing 101 blue poker chips labeled in $5 \notin$ increments, from $\$ 0$ to $\$ 5$. If the amount on the chip was equal to or greater than the player's reported selling price, the player would receive the buying price and would not play the lottery. Otherwise, the player would play the lottery and either win $\$ 5$ or nothing, depending on the outcome. Two display boards were used to explain this procedure and to demonstrate that it was in the subject's best interests to report a true, unbiased selling price, since the buying price would be determined independently of the selling price.

Lottery 2 was described next. Subjects were asked to select the lottery, either Lottery 1 or Lottery 2, that they would prefer to play, given the choice of one. Subjects then recorded their minimum selling prices for Lottery 2 . Once again, they were cautioned against reporting a biased price.

Following this, each subject recorded the reason for his or her choice between Lotteries 1 and 2, being as complete as possible. Three subjects were then randomly selected to either play their chosen lottery or accept the experimenter's buying price for that lottery. This procedure had been 
described to them before making their responses. Where applicable, the winnings were distributed at that time.

Measures. In order to test the hypothesis of the relationship between risk and ambiguity preferences, the following measures of these constructs were used. First, each subject's risk preference was determined to be one of the following (Keeney \& Raiffa, 1976): (1) risk avoiding if the cash equivalent for Lottery 1 was less than the expected outcome of Lottery $1, \$ 2.50$; (2) risk neutral if the cash equivalent was equal to $\$ 2.50$; and (3) risk seeking if the cash equivalent was greater than $\$ 2.50$. Similarly, each subject's ambiguity preference was classified. Since Lottery 2 was just as "risky" as Lottery 1 , in that the outcomes were uncertain for both lotteries, ambiguity preference was measured as a difference between the responses to the two lotteries. Specifically, subjects were classifed as (1) ambiguity avoiding if the Lottery 2 cash equivalent was less than the cash equivalent for Lottery 1 ; (2) ambiguity neutral if the cash equivalents were equal; and (3) ambiguity seeking if the Lottery 2 cash equivalent was greater than the cash equivalent for Lottery 1 . If risk and ambiguity attitudes are governed by some common factor, then we would expect a majority of subjects to have the same risk and ambiguity preference classification, for example, being both risk and ambiguity avoiding.

A more sensitive test of the relationship between risk and ambiguity attitudes is based on quantitative measures of these preferences. For each subject, a risk premium, defined as the expectation of the risky lottery, $\$ 2.50$, minus the cash equivalent for that lottery, was calculated (Pratt, 1964). A subject who was classified as risk avoiding would have a positive risk premium, with the magnitude of the premium providing a measure of the degree of risk avoidance. Intuitively, the risk premium is the amount of expected winnings that the subject is willing to relinquish in order to avoid the riskiness of the lottery. Similarly, the ambiguity premium for each subject was calculated as the Lottery 1 cash equivalent minus the Lottery 2 cash equivalent. A subject classified as ambiguity avoiding would have a positive ambiguity premium. The magnitude of the ambiguity premium provides a measure of the expected winnings that a subject is willing to relinquish in order to avoid the additional uncertainty of the ambiguous Lottery 2 . If risk and ambiguity attitudes are governed by some common factor, then we would expect risk premiums and ambiguity premiums to be positively correlated.

There is a difficulty in interpreting the correlation between the two premiums, however, which is illustrated in Fig. 2. The difficulty is that when the subject gives a price for Lottery 1 that is different than $\$ 2.50$ that is, when the risk premium is nonzero-the range for the ambiguity premium is asymmetric about zero. For example, suppose a subject says that his or her minimum selling price for Lottery 1 is $\$ 2$. This implies a 


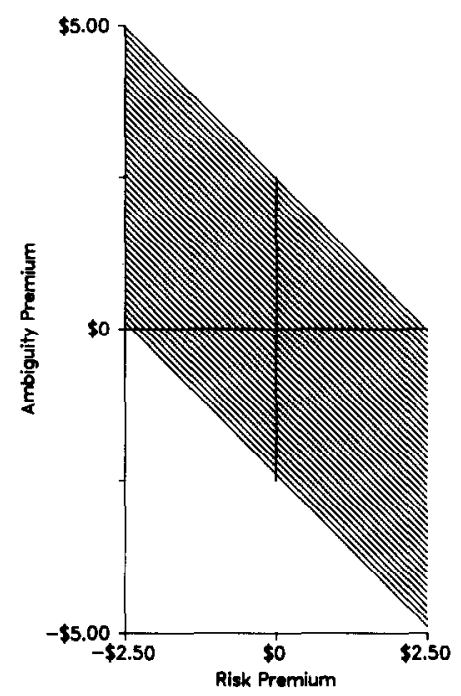

FIG. 2. Graph of the risk and ambiguity premiums for Lotteries 1 and 2 of Fig. 1. The shaded region represents the possible values of each of the premiums. Risk neutrality obtains when the risk premium is zero, and positive risk premiums imply risk-avoiding behavior. Similarly, ambiguity neutrality obtains when the ambiguity premium is zero, and positive ambiguity premiums imply ambiguity-avoiding behavior.

risk premium of $\$ 0.50$, a risk-avoiding response. As Fig. 2 indicates, given this risk premium, the ambiguity premium must lie within the interval $[-\$ 3, \$ 2]$. As a result, the subinterval within which ambiguity seeking can be expressed, $[-\$ 3, \$ 0)$, has a larger range than the subinterval within which ambiguity avoiding can be expressed, $(\$ 0, \$ 2]$. In general, these subintervals will always have unequal ranges, whenever the subject is not risk neutral.

Consequently, as indicated by the shaded region of Fig. 2, which represents the space of possible values for the two premium measures, the premiums are biased toward being negatively correlated. To compensate for this bias, a normalized ambiguity premium was also calculated for each subject. This premium was a two-piece linear transformation of the ambiguity premium to the interval $[-1,1]$, with -1 representing maximal ambiguity seeking, 1 representing maximal ambiguity avoiding, and 0 representing ambiguity neutrality.

\section{Results and Discussion}

The joint frequencies of risk preferences and ambiguity preferences are shown in Table 1. Due to several small cell sizes, the neutral and seeking 
TABLE 1

Frequencies of Risk and Ambiguity Preference Classifications (EXPERIMENT 1)

\begin{tabular}{lcccr} 
& $\begin{array}{c}\text { Risk } \\
\text { avoiding }\end{array}$ & $\begin{array}{c}\text { Risk } \\
\text { neutral }\end{array}$ & $\begin{array}{c}\text { Risk } \\
\text { seeking }\end{array}$ & Total \\
\hline Ambiguity avoiding & 7 & 4 & 6 & 17 \\
Ambiguity neutral & 1 & 2 & 2 & 5 \\
Ambiguity seeking & 3 & 0 & 1 & 4 \\
Total & 11 & 6 & 9 & 26 \\
\hline
\end{tabular}

categories were combined for statistical analysis, collapsing Table 1 into a $2 \times 2$ table. The analysis did not allow rejection of the independence of risk preferences and ambiguity preferences $\left(\chi^{2}[1]=0.03, p>.8\right)$. In addition, the correlation between risk premiums and ambiguity premiums did not achieve significance $(r[24]=-.19, p>.3)$, nor did that between risk premiums and normalized ambiguity premiums $(r[24]=.11, p>.5)$.

The results clearly fail to support the hypothesis of a general attitude toward uncertainty (Hypothesis 5) in that subjects' responses to risk and ambiguity were independent. This suggests that ambiguity avoidance is distinct from risk avoidance. One difficulty with this conclusion is the lower power of the test, which results from the relatively small sample size. This limitation is addressed in Experiment 2.

Further analyses indicated that neither risk seeking nor risk avoiding predominated, based on the mean risk premium $(t[25]=-0.23, p>.8)$, and the risk preference data in Table 1 (binomial test, $p>.8$ ). For ambiguity, avoidance predominated, as measured by either the nonnormalized $(t[25]=3.46, p<.01)$, or the normalized ambiguity premium $(t[25]=$ $3.79, p<.001)$. The choice data also support this finding, in that significantly more ambiguity-avoiding choices were made than ambiguityseeking ones (binomial test, $p<.01$ ). This result replicates those of Becker and Brownson (1964) and Yates and Zukowski (1976), in contradiction of the forced-choice hypothesis (Hypothesis 4): Using a pricing task, subjects set a lower value for the ambiguous option than for the nonambiguous option, suggesting that they would pay to avoid ambiguity.

\section{EXPERIMENT 2}

Experiment 2 was designed to both test the hostile nature explanation (Hypothesis 1) for ambiguity avoidance and provide increased statistical power for Experiment 1. The hostile nature hypothesis would predict that, among those subjects who believe that the ambiguous option is, or may be, biased against them, ambiguity avoidance would prevail. However, among those who do not believe the possibility of bias, ambiguity neutrality should obtain. The hypothesis was tested using Lotteries 1 and 
2 (Fig. 1), while forcing subjects to explicitly consider the possibility of bias in the ambiguous Lottery 2.

\section{Method}

Subjects. Two sessions were used, a manipulation session involving 20 subjects, and a control session involving 19 subjects. Subjects in both sessions were recruited from an introductory psychology class at the University of Michigan. They were not paid for their participation, but had a chance to play one of the lotteries following the experiment, and thereby possibly win $\$ 5$.

Procedure. Both the control and the manipulation conditions began with an explanation of the bag composition and playing procedure for the ambiguous game, Lottery 2 . Following this, the manipulation group engaged in a task which was omitted from the control group.

The manipulation group subjects were instructed to imagine themselves as the "managers" of Lottery 2. They were to consider how they would set the composition of Bag 2; and, particularly, if they thought it were "possible to set the composition of Bag 2 so that, no matter what color the player selects, the lottery would be biased against the player." The subjects recorded their beliefs as to the possibility of such a bias; and, then, to assure their deliberation, each recorded an explanation for his or her response. Finally, the manipulation group indicated how they would set the composition of Bag 2. This was to test for the presence of a color bias that might influence their responses.

The remainder of the procedure was the same for both the control and manipulation groups. Lottery 1 was described to the subjects. Each subject indicated a forced-choice preference for either Lottery 1 or Lottery 2 , and provided minimum selling prices for each of these lotteries. Buying prices, to be offered by the experimenter as in Experiment 1, were described, stressing the independence of their determination from the subjects' selling priccs.

Following these responses, one subject in each group was selected to play his or her preferred lottery, receiving $\$ 5$ or nothing, or to accept the buying price for that lottery, depending on his or her selling price. This procedure was explained to the subjects before their choices and minimum selling prices were requested.

\section{Results and Discussion}

No color preference was obtained in the subject-proposed compositions of Bag 2, either overall or within the bias or no-bias subgroups (e.g., $t[18]=.89$ for the overall group, $p>.3$ for all tests). Of the 20 subjects in the manipulation group, only $6(30 \%)$ believed that Bag 2 's contents could be biased against the player. More to the point, all of the 14 subjects who 
did not believe that bias was possible selected the less ambiguous Lottery 1 (binomial test, $p<.001$ ). This was an even greater extent of ambiguity avoidance than obtained among the 6 who believed that bias was possible $(66.7 \%$, binomial, $p>.6)$, which is exactly opposite the prediction of the hostile nature hypothesis. The control subjects showed an intermediate amount of ambiguity avoidance $(68.4 \%, p>.1)$. Overall, there was significant ambiguity avoidance $(79.5 \%, p<.0001)$.

As a more sensitive test, a risk premium, ambiguity premium, and normalized ambiguity premium (see Experiment 1) were calculated for each subject. The risk premiums did not differ from zero for any subgrouping of the subjects, nor did the subgroups differ from one another. Of interest in the present study are the non-normalized and normalized ambiguity premiums, which corroborated the choice data. Among those who stated that no bias was possible, the mean ambiguity premium was $.68(t[13]=$ $2.43, p<.05)$ and the mean normalized ambiguity premium was $.24(t[13]$ $=2.64, p<.05$ ), indicative of significant ambiguity avoidance. Among those who stated that bias was possible, the mean ambiguity premium was $.13(t[5]=0.19, p>.8)$ and the mean normalized ambiguity premium was $.07(t[5]=0.33, p>.7)$, indicative of ambiguity neutrality. The mean ambiguity premium among control subjects was $.42(t[18]=$ $1.59, p>.1)$, and the mean normalized ambiguity premium was .18 $(t[18]$ $=1.81, p>.05$ ). Further, these premiums did not differ significantly from the corresponding premiums of the no-bias manipulation subgroup (largest $t[24]=0.68, p>.5$ for both tests).

Thus, the hostile nature hypothesis (Hypothesis 1) was not substantiated in the present experiment. The hypothesis predicted that, among those subjects who were convinced that the ambiguous bag's contents could not be biased against them, ambiguity neutrality should have predominated. Instead, significant ambiguity avoidance obtained for these subjects. Also predicted was that the control group should have exhibited greater ambiguity avoidance than the manipulation group that claimed no possible bias. Instead, these two groups were equivalent in their ambiguity avoidance.

In addition, the present results replicate those of Experiment 1 with respect to the uncertainty avoidance hypothesis (Hypothesis 5), while adding greater statistical power to that study. Combining the data of this experiment with the data of Experiment 1, the correlations were still not significant, either between risk premiums and ambiguity premiums ( $r$ [63] $=-.15, p>.2$ ), or between risk premiums and normalized ambiguity premiums $(r[63]=.11, p>.7)$. The combined data of Experiments 1 and 2 do not indicate the presence of a general avoidance of uncertainty, of which ambiguity avoidance is a specific instance, in that there is not a significant correlation between risk avoidance and ambiguity avoidance. 


\section{EXPERIMENT 3}

Experiment 3 was designed as a test of the forced-choice hypothesis (Hypothesis 4), which proposes that subjects only avoid ambiguity because the task is a forced choice between otherwise equivalent options. Presumably, if the options were not otherwise equivalent, subjects would not avoid the ambiguous one. To test the hypothesis, four lotteries were constructed, and a choice task used. This methodology differed from that in previous studies, including Experiments 1 and 2, which used selling prices obtained in a pricing task as evidence against the forced-choice hypothesis (Becker \& Brownson, 1964; Yates \& Zukowski, 1976). Given the possibility that the choice task and pricing task may not be psychologically comparable, the present study more directly pertains to the choice situation, which is our focal concern.

Two groups of subjects participated in the study. Each group evaluated four lotteries. For Group A, two of the lotteries were Lotteries 1 and 2 in Fig. 1. Lotteries 3 and 4 were equivalent to Lotteries 1 and 2, respectively, except that the amount to be won was $\$ 4.99$, rather than $\$ 5$. The lotteries considered by Group B subjects were the same as those seen by Group A subjects, except that, for Lotteries 1 and 2 the amount to be won was $\$ 10$, rather than $\$ 5$, and for Lotteries 3 and 4 the prize was $\$ 9.99$, instead of $\$ 4.99$. The forced-choice hypothesis predicts that, for each group, both Lottery 1 and Lottery 2 should be preferred to both Lottery 3 and Lottery 4 , since the former lotteries have the higher expectation. This is a consequence of using a lexicographic decision rule, wherein the amount of ambiguity is the second dimension being considered, and expectation is the first dimension.

\section{Method: Group A}

Subjects. Sixteen University of Michigan undergraduates were recruited from a paid subject pool maintained by the Psychology Department. The experiment was presented to groups of 3-4 subjects, along with several other unrelated tasks.

Procedure. The lotteries' playing procedure was described using Lotteries 1 and 2 as examples. The subject's task was to express his or her preferences among the four lotteries. After describing the procedure, the response sheets were distributed and their graphical displays explained. The subjects were informed that none of the lotteries would actually be played; they were hypothetical. However, recall that the subjects were paid for their participation in the session, and were believed to be motivated to be reliable. Each subject ranked the four lotteries in order of decreasing preference, from 1 to 4 . 
TABLE 2

Preference ORDERINGS, AND theIr FreQuenCIES, fOR LOTTERIES 1-4

(EXPERIMENT 3)

\begin{tabular}{|c|c|c|c|c|c|}
\hline \multirow[b]{2}{*}{ Classification } & \multicolumn{4}{|c|}{ Pattern } & \multirow[b]{2}{*}{ Frequency } \\
\hline & & & & & \\
\hline $\begin{array}{l}\text { Ambiguity avoiding, } \\
\text { nonlexicographic }\end{array}$ & 1 & 3 & 2 & 4 & 35 \\
\hline $\begin{array}{l}\text { Ambiguity avoiding, } \\
\text { lexicographic }\end{array}$ & 1 & 2 & 3 & 4 & 4 \\
\hline $\begin{array}{l}\text { Ambiguity seeking, } \\
\text { nonlexicographic }\end{array}$ & 2 & 4 & 1 & 3 & 5 \\
\hline $\begin{array}{l}\text { Ambiguity seeking, } \\
\text { lexicographic }\end{array}$ & 2 & 1 & 4 & 3 & 3 \\
\hline Other & $\begin{array}{l}1 \\
2\end{array}$ & $\begin{array}{l}2 \\
1\end{array}$ & $\begin{array}{l}4 \\
3\end{array}$ & $\begin{array}{l}3 \\
4\end{array}$ & $\begin{array}{l}0 \\
0\end{array}$ \\
\hline
\end{tabular}

\section{Method: Group B}

Subjects. Thirty-one unpaid undergraduates participated in fulfillment of a requirement of the introductory psychology courses at the University. They received the task, along with several different unrelated tasks, in individual sessions.

Procedure. The procedure was identical to that for Group A, except that the four lottery displays were presented on index cards which the subject ordered according to preference. Although not paid, the subjects were given the chance to actually play several of the lotteries from the session, and were so informed before performing the experimental task.

\section{Results and Discussion}

There were no statistically reliable differences between the responses of Groups A and B, so the data are presented together. There are six plausible orderings of the four lotteries that satisfy the dominance conditions of Lottery 1 being preferred to Lottery 3 and Lottery 2 being preferred to Lottery 4 . All of the orderings subjects reported were among these six. The plausible orderings are listed in Table 2, along with their frequencies of occurrence in the sample. Also shown is the classification of each pattern as either ambiguity avoiding or ambiguity seeking, and as either lexicographic or not. The six orderings were not observed equally often $\left(\chi^{2}[5]=115.77, p<.001\right)$, the number of nonlexicographic, ambiguity-avoiding patterns being the only pattern that exceeded a chance rate of occurrence $(74.5 \%$, binomial test, $p<.001)$. Also of note, the total 
number of nonlexicographic patterns significantly exceeded the number of lexicographic patterns (binomial test, $p<.001$ ).

The results do not support the forced-choice hypothesis (Hypothesis 4) with this choice task. Subjects did not prefer both Lotteries 1 and 2 to both Lotteries 3 and 4, despite the fact that the lotteries were not identical except for ambiguity. Even in the choice task used, subjects were willing to pay to avoid ambiguity, tending to prefer both nonambiguous lotteries, 1 and 3, to the ambiguous lotteries, 2 and 4 . Thus, the present results are consistent with the pricing results using cash equivalents, as described in Experiments 1 and 2, in contradiction of the forced-choice hypothesis. ${ }^{1}$

\section{EXPERIMENT 4}

The other-evaluation hypothesis (Hypothesis 2) and the self-evaluation hypothesis (Hypothesis 3 ) were jointly tested in a $2 \times 2$ between-subjects design. The other-evaluation hypothesis proposes that subjects avoid ambiguity in anticipation of an evaluation of their decisions by other people. Thus, the hypothesis would predict a stronger reaction to ambiguity among a group of subjects whose choices are made publicly rather than privately, in that an evaluation by others is more salient and probable under conditions of public choice. The self-evaluation hypothesis proposes that a subject anticipates a future evaluation of the decision by oneself. Such an evaluation is possible only with the anticipation of relevant feedback. Consequently, the self-evaluation hypothesis implies that a stronger reaction to ambiguity should obtain among a group of subjects for whom the contents of the ambiguous bag are to be exposed, than among those for whom the contents are not to be exposed, since they are more likely to anticipate the possibilities of evaluation and regret. Lotteries 1 and 2 (Fig. 1) were used to test these hypotheses.

\section{Method}

Subjects. One hundred thirty-six undergraduates were recruited from a

\footnotetext{
1 Although not relevant for an evaluation of the forced-choice hypothesis, one could in turn ask the question: Using the choice paradigm, how much are subjects willing to pay to avoid ambiguity? The subjects in Group B were presented with additional choices which allowed an estimation of this quantity. A lower bound was determined for the mean amount that subjects were willing to give up, when Lotteries 1 and 2 involved a prize of $\$ 10$, by varying the winning prize for Lotteries 3 and 4 . Subjects, on average, would trade off $\$ 0.65$ to avoid ambiguity in this situation, indicating significant ambiguity avoidance $(t[30]=3.19$, $p<.01)$. Further details regarding the procedure of this manipulation are presented elsewhere (Curley, 1986). The value of $\$ 0.65$ is comparable to that obtained with a pricing procedure by Yates and Zukowski (1976) - $\$ 0.10$ when the prize was $\$ 1$ - and Experiments 1 and $2-\$ 0.48$ when the prize was $\$ 5$.
} 
paid subject pool operated by the Psychology Department. The experiment was conducted in groups of 3-5 subjects, along with several unrelated tasks. One subject from each group played his or her preferred lottery, receiving either $\$ 5$ or nothing, depending on the outcome.

Procedure. Two factors were manipulated in the experiment in a $2 \times 2$ design. The first factor was the other-evaluation factor and the second was the self-evaluation factor; each had a "high" and a "low" level. In the high-other-evaluation conditions, it was understood that the subject selected to play a lottery would announce his or her choice and play that lottery in front of the entire group of subjects-publicly. In the lowother-evaluation conditions, it was understood that the selected subject would remain after the experiment and play the lottery after the others had left. In the high-self-evaluation conditions, the contents of the ambiguous bag, the bag for Lottery 2 , were exposed immediately after the subject played his or her chosen lottery. In the low-self-evaluation conditions, it was made clear that the contents of the ambiguous bag would never be known to any of the subjects.

All four experimental conditions began with identical descriptions of Lotteries 1 and 2. Following this, in the low-self-evaluation conditions only, it was explained that the contents of the ambiguous bag would never be known to the subjects, not even after the experiment.

The playing procedure was then presented, a different display board and playing procedure being used for each of the four conditions of the design. The summaries of the playing procedures, as they appeared on the display boards, appear in Table 3. In all conditions, the first two steps of the procedure were for each subject to respond and then for one of the subjects in that group to be selected at random.

The remaining steps of the playing procedures differed among the four conditions. As Table 3 shows, in the high-other-evaluation conditions, the lottery was to be played immediately after the player was selected. In the low-other-evaluation conditions, the playing of the lottery was to be delayed until the experiment's end, after the other subjects had left. After the lottery was to be played, the high-self-evaluation conditions required the exposure of the contents of the ambiguous bag. The contents were to be shown to everyone in the high-self-evaluation/high-other-evaluation condition, and only to the player in the high-self-evaluation/low-otherevaluation condition, since only that subject would be present in the latter case. This verification of the ambiguous bag's contents was explicitly omitted in the low-self-evaluation conditions; instead, the subjects were reminded of the fairness of the game as guaranteed by the playing procedure.

After having the applicable playing procedure clarified, the subjects received their response sheets and indicated which of the two lotteries 
TABLE 3

Summaries of the Playing Procedures by ExPERIMENTAL Condition (EXPERIMENT 4)

\begin{tabular}{|c|c|c|}
\hline & High self-evaluation & Low self-evaluation \\
\hline $\begin{array}{l}\text { High } \\
\text { other- } \\
\text { evaluation }\end{array}$ & $\begin{array}{l}\text { 1. You choose either } \\
\text { Lottery } 1 \text { or Lottery } 2 \\
\text { 2. One person is selected } \\
\text { randomly to play a } \\
\text { lottery at that time } \\
\text { 3. That person plays } \\
\text { his/her chosen lottery } \\
\text { and receives the payoff } \\
\text { of either } \$ 5 \text { or nothing } \\
\text { 4. The bag for Lottery } 2 \\
\text { is emptied for } \\
\text { everyone to see }\end{array}$ & $\begin{array}{l}\text { 1. You choose either } \\
\text { Lottery } 1 \text { or Lottery } 2 \\
\text { 2. One person is selected } \\
\text { randomly to play a } \\
\text { lottery at that time } \\
\text { 3. That person plays } \\
\text { his/her chosen lottery } \\
\text { and receives the payoff } \\
\text { of either } \$ 5 \text { or nothing }\end{array}$ \\
\hline $\begin{array}{l}\text { Low } \\
\text { other- } \\
\text { evaluation }\end{array}$ & $\begin{array}{l}\text { 1. You choose either } \\
\text { Lottery } 1 \text { or Lottery } 2 \\
\text { 2. One person is selected } \\
\text { randomly to remain } \\
\text { after everyone else } \\
\text { has left } \\
\text { 3. That person plays } \\
\text { his/her chosen lottery } \\
\text { and receives the payoff } \\
\text { of either } \$ 5 \text { or nothing } \\
\text { 4. The bag for Lottery } 2 \\
\text { is emptied for } \\
\text { that person to see }\end{array}$ & $\begin{array}{l}\text { 1. You choose either } \\
\text { Lottery } 1 \text { or Lottery } 2 \\
\text { 2. One person is selected } \\
\text { randomly to remain } \\
\text { after everyone else } \\
\text { has left } \\
\text { 3. That person plays } \\
\text { his/her chosen lottery } \\
\text { and receives the payoff } \\
\text { of either } \$ 5 \text { or nothing }\end{array}$ \\
\hline
\end{tabular}

they preferred. This was a forced-choice task. A strength of preference was then rated on a scale with anchors identified with the two lotteries. Ratings were used so as to avoid the difficulty of the asymmetry of the ambiguity premium as a measure of ambiguity avoidance, as was described in the context of Experiment 1. The ratings were coded on a scale of 1 to 7 in the analyses, with 1 representing maximal ambiguity seeking, 4 representing ambiguity neutrality, and 7 representing maximal ambiguity avoiding. Finally, the responses were collected and the appropriate playing procedure was enacted as described.

\section{Results and Discussion}

No effect was found for differing group sizes on either the ratings or the choices. The percentage of ambiguity-avoiding choices and the mean strength of preference rating for each condition are shown in Fig. 3. A multidimensional test of independence, applied to the choice data, re- 

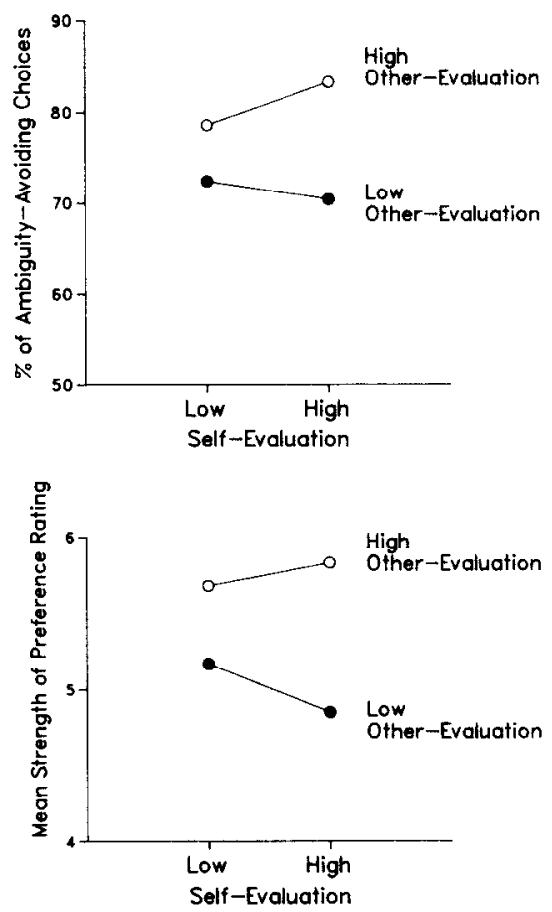

FIG. 3. Percentage of ambiguity-avoiding choices and mean strength of preference rating for the four experimental conditions of Experiment 4. A higher rating indicates greater ambiguity avoidance.

vealed no significant dependencies $\left(\chi^{2}[4]=1.67, p>.7\right)$. However, as Fig. 3 indicates, the pattern of the results was identical for the choice and rating data. A two-way analysis of variance applied to the latter indicated a significant effect of the other-evaluation factor $(F[1,110]=6.14, p<$ $.05)$; but not significant were the self-evaluation factor $(F[1,110]=0.08$, $p>.7)$ and the interaction of these factors $(F[1,110]=0.63, p>.4)$. All four conditions exhibited significant ambiguity avoidance, both in the choice data (binomial test, $p<.05$ for all tests) and in the rating data (minimum $t[26],=2.79, p<.01$ for all tests, smallest $\mathrm{df}=26$ ).

Clearly, the present experiment indicates no support for the self-evaluation hypothesis (Hypothesis 3 ). Those subjects who could anticipate the revelation of the contents of the ambiguous bag, for whom regret was possible, did not exhibit greater ambiguity avoidance than those subjects in the low-self-evaluation conditions, for whom the feedback necessary for regret was not anticipated. On the other hand, the other-evaluation hypothesis (Hypothesis 2) was sustained. Publicizing subjects' decisions, 
which increased the likelihood of the evaluation of the decisions by others in the groups, significantly raised the level of ambiguity avoidance.

That ambiguity avoidance obtained even for the low-other-evaluation conditions might suggest that at least one other source of ambiguity a'voidance must be present. Although this may well be possible, the existence of ambiguity avoidance in these conditions is not sufficient proof of this conjecture. Note that even the low-other-evaluation conditions have the necessary presence of the experimenter. The experimenter is a significant other to most subjects. So, the experimenter's presence may covertly influence a decision maker to behave in a manner that he or she believes will appear competent. Thus, the significant extent of ambiguity avoidance found even in the low-other-evaluation conditions is to be expected under the other-evaluation hypothesis.

\section{EXPERIMENT 5}

Experiment 5 was designed as a test of the mistake hypothesis. The mistake hypothesis claims that in avoiding ambiguity subjects are exhibiting a nonconscious bias. The prediction was that, if the situation were sufficiently understood, subjects would correct this bias. The procedure used was similar to that described by Slovic and Tversky (1974), and may be regarded as a replication of their study using different lotteries. The present experiment employed Lotteries 1 and 2 (Fig. 1), with slight variations as described below. If a subject's ambiguity avoidance is due to a nonconscious bias, as the mistake hypothesis indicates, then the subject should react to the presentation of prescriptive counterarguments by altering his or her preferences.

\section{Method}

Subjects. Seventy undergraduates at the University of Michigan were recruited from several introductory psychology courses. The subjects were separated into three groups, each of which had the same tasks. They were not paid for their participation, but understood that one of them would be selected to play one of the lotteries in the experiment, receiving the outcome of that lottery.

Procedure. The subjects' tasks were to indicate their preferences among four bets. The bets were lotteries that were derived from Lotteries 1 and 2 . There were three differences between these bets and the lotteries in Fig. 1: (a) The winning amount was $\$ 10$, rather than $\$ 5$; (b) the chip colors used were blue and red, rather than white and red; and (c) the winning color was specified in the bet, rather than by the subject. The latter difference implied four bets from the two lotteries. In Bet Red 1, the subject was to select a chip at random from the nonambiguous Bag 1 
and receive $\$ 10$ if the chip was red, nothing if the chip was blue. In Bet Blue 1, the subject selected a chip at random from Bag 1, and received $\$ 10$ for a blue chip, nothing for a red chip. Bet Red 2 and Bet Blue 2 were similarly defined using the ambiguous Bag 2 for the random drawing, with the winning color as specified.

The bets were described in the first of two response booklets that were used. The subject's task was to choose between bets. A pair of bets was offered, beginning with Bet Red 2 versus Bet Blue 2, and subjects indicated which they would prefer, or if they were indifferent. Thus, this was not a forced-choice task. Subjects then rated their strength of preference on a scale with endpoints labcled with the names of the two bets, and with the center labeled "Indifferent." The strength of preference ratings were coded on a scale of 1 to 5 in the analyses. Following their responses to Bets Red 2 and Blue 2, the subjects responded to three more bet pairs: Bet Red 1 versus Bet Blue 1, Bet Red 1 versus Bet Red 2, and Bet Blue 1 versus Bet Blue 2 . Note that each of the first two bet pairs involved the same bag. These comparisons tested for the existence of a color preference between red and blue. The second two bet pairs involved the same color, but different bags; choices involving these bets tested for an ambiguity preference.

After the four sets of responses were elicited, the first response booklet was collected and the second one distributed. The purpose of the second booklet was to present arguments designed to influence subjects' choices, to allow subjects to reconsider their stated preferences, and then to determine if their choices changed. The booklet described each of the bets, outlined the choices it required, and summarized arguments for two patterns of response to the last two bet pairs in the first booklet. It was these last two choice pairs that were repeated in the second booklet. The first response pattern was that of either ambiguity seeking or ambiguity avoidance; the second response pattern was that of ambiguity neutrality. The rationales given to the subject for the first response pattern argued for a consistent preference either for the nonambiguous bag in both choices (Pattern la) or for the ambiguous bag in both choices (Pattern 1b). That is, Pattern 1 argued that subjects should react to ambiguity, either by avoiding it or seeking it. The rationale for the second response pattern presented the argument of statistical decision theory for indifference between the ambiguous and nonambiguous bags (Pattern 2). That is, Pattern 2 argued that subjects should not react to ambiguity, and should be indifferent between the lotteries in each pair. The rationales, as presented to the subjects, are described in more detail in the Appendix.

Following the presentation of the arguments, subjects were asked to reconsider the last two choices from the first response hooklet. They were assured that they were not compelled to either change or stick to 
their original decisions. After the responses were made, a subject from each session was chosen at random. One of the bet pairs was randomly selected, and the subject played the lottery for which he or she had indicated a preference, receiving the outcome of that lottery. This procedure had been explained to the subjects before the responses were requested.

\section{Results and Discussion}

The subjects did not exhibit any systematic color preferences, either in their responses to the first two bet pairs in the first response booklet, or in their response patterns to the remaining four bet pairs. We concentrate, then, on the latter four bet pairs as tests of the mistake hypothesis.

As Table 4 shows, only five times was there a switch from an ambiguity preference to ambiguity neutrality after the rationales were presented. One subject accounted for two of these switches, and three subjects made this switch only for the first of the two bet pairs. Furthermore, an almost equal number of times - four times-the opposite switch occurred; again with one subject providing two of these switches. Clearly, subjects tended to persist in their choices despite the arguments presented, an observation that attained statistical significance (smaller $\chi^{2}[1]$ $=18.64, p<.0001$ for each subtable in Table 4, after combining the neutral and seeking categories to obtain adequate cell sizes). Also observed was that subjects tended to avoid, rather than seek, ambiguity in both the first presentations $(z=3.84, n=140, p<.001)$ and the second presentations $(z=3.07, n=140, p<.01)$, in agreement with previous studies.

The strength of preference ratings corroborated the choice data. The mean ratings for both bet pairs and both presentations are shown in Table 5. This table also contains the mean difference between the first and second presentations of a nonindifference measure, NI. For each subject for each bet pair, this measure was calculated as

$$
N I=\mid \text { Rating }-3.00 \mid \text {. }
$$

This value measures the extent to which the subject is not indifferent between the bets in that pair, irrespective of the direction of preference. Since indifference, which is expressed by a rating of 3.00 , is the normatively predicted response, this measure is an index of subjects' disagreement with the normative model of statistical decision theory. As Table 5 indicates, there is significant ambiguity avoidance in both presentations, and there is no significant decrease in subjects' nonindifference to ambiguity between presentations as a result of exposure to arguments.

The reluctance of subjects to change their behavior after the presentation of a prescriptive counterargument in the present choice situation replicates the result which has been obtained in other choice situations in- 


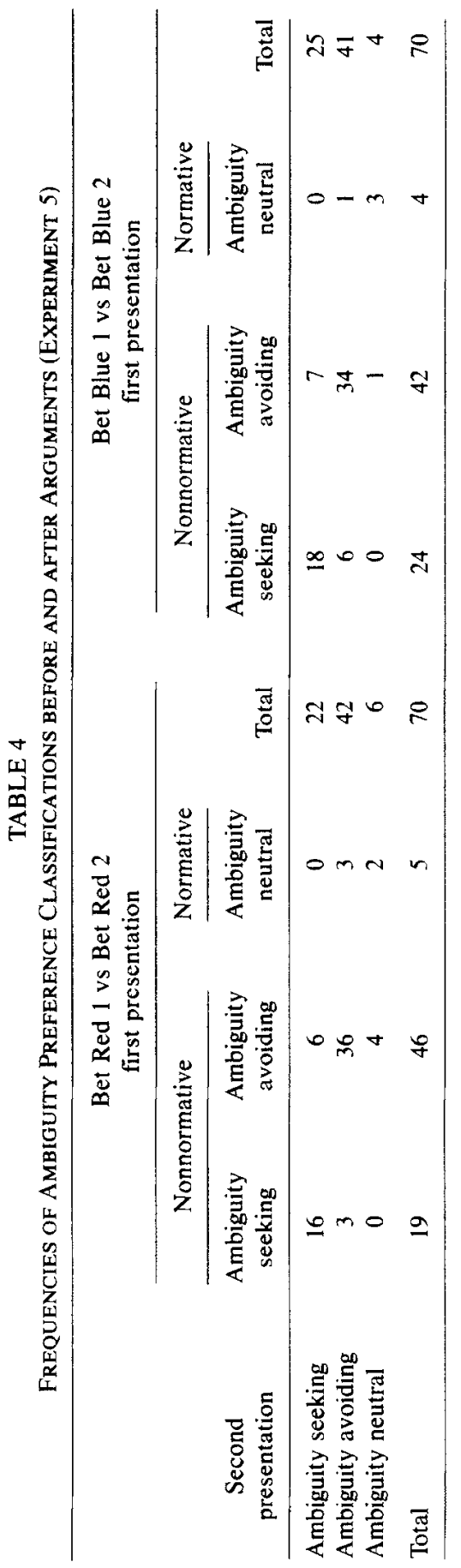


volving ambiguity (MacCrimmon, 1968; MacCrimmon \& Larsson, 1979; Slovic \& Tversky, 1974). The finding is in contrast to the prediction of the mistake hypothesis that subjects would not avoid ambiguity after the "mistake" was pointed out to them. It is not the case that subjects, upon reflection, will "correct" themselves after their "error" is highlighted and explained.

\section{GENERAL DISCUSSION}

The present line of experiments has moved toward filling a gap in the research on ambiguity. Our concern was with the question, "Why is ambiguity avoided?" We noted that, although numerous explanations have been advanced, the empirical support for each of these hypotheses was sparse. Given the prevalence of ambiguity in real-world decision situations, a better understanding of people's reactions to ambiguity was desired, and the present experiments have succeeded in advancing that goal. In particular, only one of the explanations that have been proposed, the other-evaluation hypothesis (Hypothesis 2), was supported. Further, the experiments verified the existence of the phenomenon of ambiguity avoidance, with approximately $70 \%$ of the subjects choosing the less ambiguous option overall, thereby exhibiting the characteristic ambiguityavoiding behavior.

On the basis of the present findings, the phenomenon of ambiguity avoidance is not attributable to a belief that the outcome-determination process is "hostile" (Hypothesis 1). It is not attributable to an antici-

TABLE 5

Mean Strength of Preference Ratings and Mean Nonindifference Measure (NI) DIFFERENCE (EXPERIMENT 5)

\begin{tabular}{lcc}
\hline & $\begin{array}{c}\text { Bet Red 1 } \\
\text { vs } \\
\text { Bet } \operatorname{Red~2}\end{array}$ & $\begin{array}{c}\text { Bet Blue 1 } \\
\text { vs } \\
\text { Bet Blue 2 }\end{array}$ \\
\hline $\begin{array}{c}\text { Mean rating, } \\
\text { first presentation } \\
\text { Mean rating, } \\
\quad \text { second presentation }\end{array}$ & $3.61^{* *}$ & $3.41^{*}$ \\
$\begin{array}{c}\text { Mean NI difference, } \\
\text { first-second }\end{array}$ & $3.34^{*}$ & $3.36^{*}$ \\
\hline
\end{tabular}

a Compared to $3=$ ambiguity neutrality, $1=$ maximal ambiguity seeking, and $5=\max$ imal ambiguity avoiding.

$* p<.05$.

$* * p<.001$. 
pated future self-evaluation of the decision (Hypothesis 3). It is not due to the eixstence of a lexicographic decision rule operating in a forcedchoice task (Hypothesis 4). It is not related to a general avoidance of uncertainty of the type described by risk-aversion measures (Hypothesis 5). And it is not a systematic error that subjects are willing to correct upon proper understanding (Mistake hypothesis).

The phenomenon appears to result from an anticipation by decision makers that their decisions will be evaluated by others. The present experiment used only implicit evaluation, in that others merely observed the decision, but neither commented on it, nor concretely rewarded or punished it. Nevertheless, an effect was oblained. Thus, the decision maker avoids ambiguity in the belief that such a choice is most justifiable to others, that is, most socially acceptable.

One limitation of the experiments leading to this finding is that many of them resulted in null conclusions, i.e., in the failure to reject the null hypotheses. As we stated, this was to be expected on the basis of the present state of knowledge regarding the sources of ambiguity reactions. Although not consistent with common practice in the reporting of experimental results, we believe the null findings to be at least as important as those rejecting the null hypotheses, an argument which has been presented in detail by Greenwald (1975). Further, the validity of the null results is substantiated by the rejection of the null hypothesis in Experiment 4 , and in the replication of the phenomenon of ambiguity avoidance throughout the experiments. In summary, the experiments support the psychological interpretation of other-evaluation as a feasible hypothesis both in that Experiment 4 showed an effect of manipulating an other-evaluation factor, and in that no other proposed hypothesis was similarly substantiated.

One interesting issue with respect to the other-evaluation hypothesis is why people would perceive an ambiguity-avoiding response as more justifiable. A possibility is that subjects do so because they evaluate decisions on the basis of decision outcomes, and not on the basis of the decision process. Accordingly, they anticipate that their own decisions will be so evaluated, and choose the nonambiguous option to avoid the outcome of losing with an ambiguous bag that contains fewer than 50 winning chips.

Another point of interest, mentioned earlier, is the normative issue surrounding ambiguity reactions, specifically, is ambiguity avoidance reasonable? In that much of our behavior is evaluated by others, the anticipation of this evaluation in making a choice is not necessarily irrational. Thus, further investigation is warranted to illuminate this issue, possibly leading to the incorporation of ambiguity reactions into normative theories of choice. 


\section{APPENDIX: ARGUMENTS PRESENTED TO SUBJECTS IN EXPERIMENT 5}

The following arguments were verbally presented to the subjects in Experiment 5; written summaries of the arguments were also included in the second response booklet, for referral while considering the choices required:

Two common response patterns are offered by people faced with the last two choices that you considered in the first response booklet. Rationales for each of these response patterns are now reviewed.

\section{Pattern 1}

In Decision Pattern 1, the person always chooses to bet on either Bag 1 or Bag 2. The person argues that it is inappropriate to be indifferent between Bet Red 1 and Bet Red 2 because it is unlikely that the two bags have the same composition. Similarly, it is inappropriate to be indifferent between Bet Blue 1 and Bet Blue 2 because the two bags are unlikely to have the same composition. Therefore, one of the following subpatterns should be used.

In Pattern la, the person always prefers Bag 1 over Bag 2. Thus, the person chooses Bet Red 1 over Bet Red 2 because the number of red chips in Bag 2, being unspecified, might very well be less than 50 , the number of red chips in Bag 1. Similarly, the person chooses Bet Blue 1 over Bet Blue 2 because the number of blue chips in Bag 2, being unspecified, might be less than 50, the number of blue chips in Bag 1 .

In Pattern 1b, the person always prefers Bag 2 over Bag 1 . Thus, the person chooses Bet Red 2 over Bet Red 1 because the number of red chips in Bag 2, being unspecified might be more than 50, the number of red chips in Bag 1. Similarly, the person chooses Bet Blue 2 over Bet Blue 1 because the number of blue chips in Bag 2, being unspecified, might be more than 50, the number of blue chips in Bag 1. In either case, indifference is inappropriate, and one should always prefer drawing from either one bag or the other.

\section{Pattern 2}

In Decision Pattern 2, the person is indifferent between drawing from Bag 1 and drawing from Bag 2. The person argues that it is inappropriate to prefer one bag or the other. The rationales against a preference for either bag follow.

Consider Pattern 1a. Suppose the person chooses Bet Red 1 over Bet Red 2. This implies that he or she thinks that there are fewer than 50 red chips in Bag 2, since Bag 1 contains 50 red chips for certain. Similarly, if the person chooses Bet Blue 1 over Bet Blue 2, this implies that he or she thinks that there are fewer than 50 blue chips in Bag 2, since Bag 1 con- 
tains 50 blue chips for certain. But this is a contradiction. Bag 2 cannot both have less than 50 red chips and less than 50 blue chips.

Now consider Pattern lb. If the person chooses Bet Red 2 over Bet Red 1, this implies that he or she thinks that there are more than 50 red chips in Bag 2, since Bag 1 contains 50 red chips for certain. Similarly, if the person chooses Bet Blue 2 over Bet Blue 1, this implies that he or she thinks that there are more than 50 blue chips in Bag 2, since Bag 1 contains 50 blue chips for certain. Again, this is a contradiction. Bag 2 cannot both have more than 50 red chips and more than 50 blue chips.

In either case, a preference is inappropriate, and one should be indifferent between drawing from either one bag or the other.

\section{REFERENCES}

Becker, G. M., DeGroot, M. H., \& Marschak, J. (1964). Measuring utility by a single-response sequential method. Behavioral Science, 9, 226-232.

Becker, S. W., \& Brownson, F. O. (1964). What price ambiguity? Or the role of ambiguity in decision-making. Journal of Political Economy, 72, 62-73.

Bell, D. E. (1982). Regret in decision making under uncertainty. Operations Research, 30. $961-981$.

Bell, D. E. (1983). Risk premiums for decision regret. Management Science, 29, $1156-1166$.

Curley, S. P. (1986). Descriptive models of choice under ambiguity. Doctoral dissertation, University of Michigan, Ann Arbor.

Curley, S. P., Eraker, S. A., \& Yates, J. F. (1984). An investigation of patients' reactions to therapeutic uncertainty. Medical Decision Making, 4, 501-511.

Curley, S. P., \& Yates, J. F. (1985). The center and range of the probability interval as factors affecting ambiguity preferences. Organizational Behavior and Human Decision Processes, 36, 273-287.

DeGroot, M. H. (1970). Optimal statistical decisions. New York: McGraw-Hill.

Einhorn, H. J., \& Hogarth, R. M. (1985). Ambiguity and uncertainty in probabilistic inference. Psychological Review, 92, $433-461$.

Ellsberg, D. (1961). Risk, ambiguity, and the Savage axioms. Quarterly Journal of Economics, 75, 643-669.

Ellsberg, D. (1963). Risk, ambiguity, and the Savage axioms: Reply. Quarterly Journal of Economics, 77, 336-342.

Fellner, W. (1961). Distortion of subjective probabilities as a reaction to uncertainty. Quarterly Journal of Economics, 75, 670-689.

Fishburn, P. C. (1980). A simple model for the utility of gambling. Psychomerrika, 45 , $435-448$.

Freedman, D. A., \& Purves, R. A. (1969). Bayes' method for bookies. Annals of Mathematical Statistics, 40, 1177-1186.

Gardenfors, P. (1979). Forecasts, decisions and uncertain probabilities. Erkenntnis, 14, $159-181$.

Goldsmith, R. W., \& Sahlin, N. E. (1982). The role of secondary probabilities in decision making. In P. C. Humphreys, O. Svenson, \& A. Vari (Eds.), Analysing and aiding decision processes. Amsterdam: North-Holland.

Goldstein, W. M. (1984). The relationship between judgment and choice. Doctoral dissertation, University of Michigan, Ann Arhor

Greenwald, A. G. (1975). Consequences of prejudice against the null hypothesis. Psychological Bulletin, 82, 1-20. 
Grether, D. M., \& Plott, C. R. (1979). Economic theory of choice and the preference reversal phenomenon. American Economic Review, 69, 623-638.

Hamm, R. M., \& Bursztajn, H. (1979, September). A medical version of a decision theory paradox: Is it still a paradox? Paper presented at the First Annual Meeting of the Society for Medical Decision Making, Cincinnati, $\mathrm{OH}$.

Hogarth, R. M., \& Kunreuther, H. (1984). Risk, ambiguity, and insurance. Unpublished manuscript, University of Chicago, Center for Decision Research.

Hogarth, R. M., \& Kunreuther, H. (1985). Ambiguity and insurance decisions. The American Economic Review. Papers and Proceedings of the 97th Annual Meeting of the American Economic Association, 75, 386-390.

Keeney, R. L., \& Raiffa, H. (1976). Decisions with multiple objectives: Preferences and value tradeoffs, New York: Wiley.

Keynes, I. M. (1921). A treatise on probability. London: Macmillan Co.

Knight, F. H. (1921). Risk, uncertainty and profit. Boston: Houghton Mifflin.

Langer, E. J. (1975). The illusion of control. Journal of Personality and Social Psychology, 32, 311-328.

Lichtenstein, S., \& Slovic, P. (1971). Reversals of preference between bids and choices in gambling decisions. Journal of Experimental Psychology, 89, 46-55.

Luce, R. D., \& Raiffa, H. (1957). Games and decisions. New York: Wiley.

MacCrimmon, K. R. (1968). Descriptive and normative implications of the decision-theory postulates. In K. Borch \& J. Mossin (Eds.), Risk and uncertainty. New York: Macmillan Co.

MacCrimmon, K. R., \& Larsson, S. (1979). Utility theory: Axioms versus 'paradoxes.' In M. Allais \& O. Hagen (Eds.), Expected utility hypotheses and the Allais paradox. Boston: Reidel.

Pratt, J. W. (1964). Risk aversion in the small and in the large. Econometrica, 32, 122-136.

Raiffa, H. (1961). Risk, ambiguity, and the Savage axioms: Comment. Quarterly Journal of Economics, 75, 690-694.

Roberts, H. V. (1963). Risk, ambiguity, and the Savage axioms: Comment. Quarterly Journal of Economics, 77, 327-336.

Savage, L. J. (1972). The foundations of statistics (2nd rev. ed.). New York: Dover.

Slovic, P. (1975). Choice between equally valued alternatives. Journal of Experimental Psychology: Human Perception and Performance, 1, 280-287.

Slovic, P., Fischhoff, B., \& Lichtenstein, S. (1982). Response mode, framing, and information-processing effects in risk assessment. In R. Hogarth (Ed.), New directions for methodology of social and behavioral science: Question framing and response consistency. San Francisco: Jossey-Bass.

Slovic, P., \& Tversky, A. (1974). Who accepts Savage's axiom? Behavioral Science, 19, 368-372.

Toda, M., \& Shuford, E. H., Jr. (1965). Utility, induced utilities, and small worlds. Behavioral Science, 10, 238-254.

Tversky, A. (1969). Intransitivity of preferences. Psychological Review, 76, 31-48.

Tversky, A., \& Kahneman, D. (1983). Extensional versus intuitive reasoning: The conjunction fallacy in probability judgment. Psychological Review, 90, 293-315.

von Neumann, J., \& Morgenstern, O. (1953). Theory of games and economic behavior (3rd ed.). Princeton, NJ: Princeton Univ. Press.

Winkler, R. L. (1972). Introduction to Bayesian inference and decision. New York: Holt, Rinehart \& Winston.

Yates, J. F, \& Zukowski, L. G. (1975). The anatomy and consequences of ambiguity in decision making (Tech. Rep. MMPP 75-2). Ann Arbor, MI: Michigan Mathematical Psychology Program, University of Michigan.

Yates, J. F., \& Zukowski, L. G. (1976). Characterization of ambiguity in decision making. Behavioral Science, 21, 19-25.

ReCeIVED: May 20, 1985 